\title{
ANÁLISES QUALI- E QUANTITATIVA DE CAFÉS COMERCIAIS VIA RESSONÂNCIA MAGNÉTICA NUCLEAR
}

\author{
Leila Aley Tavares e Antonio Gilberto Ferreira*
}

Departamento de Química, Universidade Federal de São Carlos, CP 676, 13560-970 São Carlos - SP, Brasil

Recebido em 18/3/05; aceito em 4/11/05; publicado na web em 18/5/06

\begin{abstract}
QUALI- AND QUANTITATIVE ANALYSIS OF COMMERCIAL COFFEE BY NMR. Coffee is one of the beverages most widely consumed in the world and the "cafezinho" is normally prepared from a blend of roasted powder of two species, Coffea arabica and Coffea canephora. Each one exhibits differences in their taste and in the chemical composition, especially in the caffeine percentage. There are several procedures proposed in the literature for caffeine determination in different samples like soft drinks, coffee, medicines, etc but most of them need a sample workup which involves at least one step of purification. This work describes the quantitative analysis of caffeine using ${ }^{1} \mathrm{H}$ NMR and the identification of the major components in commercial coffee samples using 1D and 2D NMR techniques without any sample pre-treatment.
\end{abstract}

Keywords: caffeine; quantitative ${ }^{1} \mathrm{H}$ NMR; coffee composition.

\section{INTRODUÇÃOO}

O café é uma das bebidas mais consumidas mundialmente e a sua importância econômica tem incentivado vários estudos, principalmente no que se refere à composição química do grão e da bebi$\mathrm{da}^{1-7}$. O teor de cafeína no café apresenta uma variação que depende, dentre outros fatores, da espécie da planta utilizada, sendo que a Coffea arabica (arábica) contém em média $1 \%$ do alcalóide e a Coffea canephora (robusta) em torno de $2 \%^{8-10}$. Entre os compostos mais estudados a cafeína é sem dúvida alguma o alcalóide, extraído do café, mais conhecido e também o que se encontra em maior quantidade, embora muitos outros compostos estejam presentes.

Outra substância presente no café e que tem despertado interesse é a trigonelina, importante não pela sua presença em si, mas principalmente, pelos produtos da sua degradação que podem ser gerados no processo de torra. Por isso, o grau de torra do café, tempo de torra versus temperatura, pode ser estimado fazendo-se a relação do teor de trigonelina com o do ácido nicotínico resultante da sua degradação térmica ${ }^{8}$. O ácido nicotínico ou niacina é uma importante vitamina para o metabolismo humano, podendo ser encontrado em quantidades que chegam próximo a $20 \mathrm{mg}$ para cada $100 \mathrm{~g}$ de café torrado dependendo, evidentemente, do grau de torra do café $^{11}$ e, portanto, a sua quantificação é de suma importância.

A temperatura de torra tem sido fortemente associada ao aroma do café. Durante a torrefação, reações de Maillard, degradação de Stecker, pirólise e outras reações químicas produzem um grande número de diferentes compostos voláteis, sendo que mais de 800 compostos de várias classes já foram identificados. Além disso, o grau de torra tem impacto na concentração de vários compostos importantes, como por ex., guaiacol e 2-furfuriltiol, que aumentam com o grau de torra, enquanto que a 2,3-butanodiona e 2,3-pentanodiona apresentam um máximo de concentração para um grau de torra médio e exibem concentração mínima em cafés com alto grau de torra ${ }^{4}$.

Durante o processo de torrefação também se produzem outros compostos derivados de piridinas, pirróis e alguns compostos bicíclicos, os quais têm grande contribuição para o sabor final da bebida $^{8,12,13}$.

*e-mail: giba_04@yahoo.com.br
Além desses, outros compostos de grande interesse são os ácidos clorogênicos, que têm chamado atenção devido à sua atividade anticancerígena comprovada para alguns tipos de câncer $^{14}$, e a sua ação benéfica no combate a doenças cardiovasculares devido às suas propriedades antioxidantes ${ }^{15}$. Eles são formados principalmente pela esterificação do ácido quínico com os ácidos cafeico, ferúlico ou pcumárico. A esterificação também pode ocorrer entre o ácido quínico e dois ácidos cafeicos, ou ainda, entre o ácido quínico com um ácido cafeico e um ferúlico. $\mathrm{O}$ teor total desses ácidos nos grãos verdes de café é de aproximadamente 6,0 e 9,5\% $\left(\mathrm{m} \mathrm{m}^{-1}\right)$ para as espécies arábica e robusta, respectivamente ${ }^{16}$, muito embora se encontrem somente traços dos ácidos livres nos grãos de café verdes ${ }^{8}$. Os principais isômeros dos ácidos clorogênicos foram identificados e quantificados, recentemente, em amostras de cafés comerciais, onde se observou para todas as amostras uma predominância dos ácidos cafeoilquínicos com cerca de 77 a $81 \%$ do total de ácidos clorogênicos ${ }^{11}$.

$\mathrm{Na}$ identificação dos compostos presentes no café, várias técnicas espectroscópicas são utilizadas, tanto em separado, como em associação, tais como cromatografia líquida de alta resolução $0^{7,17}$, espectroscopia nas regiões do infravermelho ${ }^{18,19}$ e infravermelho próximo $^{20}$, cromatografia gasosa ${ }^{21,22}$, cromatografia gasosa de alta resolução acoplada à espectrometria de massas ${ }^{12,23}$ e ressonância magnética nuclear ${ }^{24,25}$. A RMN tem a vantagem de não requerer nenhum pré-tratamento da amostra quando comparada às outras técnicas e, além disso, uma análise espectral detalhada de um único espectro de hidrogênio poderá fornecer informações sobre a estrutura e a composição química dos principais constituintes químicos majoritários presentes na amostra.

A RMN tem sido, em geral, utilizada na análise de cafés para identificação de substâncias isoladas ou que constituem um pequeno grupo de substâncias em uma fração extraída do café, como por ex., a investigação dos compostos poliméricos da fração espumante de cafés expressos recém-preparados ${ }^{26}$, o estudo dos produtos de degradação térmica da trigonelina em sistemas modelo ${ }^{27} \mathrm{e}$ as suas quantificações em amostras de cafés torrados ${ }^{28} \mathrm{e}$, também, na identificação de polissacarídeos da fração aquosa de cafés torrados ${ }^{3} \mathrm{e}$ dissacarídeos isolados de cafés verdes ${ }^{29}$, dentre outros. No entanto, a aplicação dessa técnica na análise de amostras de cafés sem nenhum pré-tratamento ${ }^{24}$, assim como ocorre neste trabalho, ainda é muito pouco explorada. 
Neste trabalho a técnica de RMN foi aplicada utilizando-se amostras de "cafezinho", sem nenhum processo de tratamento e com dois propósitos. O primeiro foi identificar os compostos majoritários encontrados na bebida preparada a partir de pós de cafés comerciais, e o segundo, quantificar os teores de cafeína tanto nos cafés preparados em laboratório como nos encontrados em estabelecimentos comerciais da cidade de São Carlos-SP.

\section{PARTE EXPERIMENTAL}

Todos os espectros de RMN foram obtidos em um equipamento de 9,4 T (400,13 MHz para frequiência do hidrogênio), marca Bruker, modelo DRX400, a uma temperatura de $303 \mathrm{~K}$ e equipado com uma sonda de detecção inversa de $5 \mathrm{~mm}$.

\section{Preparação das amostras}

As amostras de pós de cafés torrados utilizadas na identificação dos constituintes majoritários foram preparadas de modo a se obter uma infusão concentrada utilizando-se cerca de $40 \mathrm{~g}$ de pó e $250 \mathrm{~mL}$ de água fervente, aproximadamente $95^{\circ} \mathrm{C}$, que foi adicionada sobre os pós e filtrados em uma cafeteira manual, usando-se papel de filtro comum.

\section{Espectroscopia de RMN de ${ }^{1} \mathbf{H}$}

Em uma alíquota de $0,6 \mathrm{~mL}$ dessa solução foram adicionadas 2 gotas de DMSO- $\mathrm{d}_{6}$. Os espectros de RMN de ${ }^{1} \mathrm{H}$ foram obtidos utilizando-se a sequiência de pulsos zgcppr (pré-saturação do sinal de HDO utilizando-se pulsos compostos), a qual se mostrou a mais eficiente dentre aquelas testadas: zgpr, noesypr1d (ambas com présaturação por ondas contínuas de baixa potência) e WATERGATE ${ }^{30}$. Além dos espectros de hidrogênio, também foram realizados os experimentos de ${ }^{13} \mathrm{C}\left\{{ }^{1} \mathrm{H}\right\}$, gCOSY, gHSQC e gHMBC, com o intuito de identificar o maior número de compostos. No entanto, para facilitar a confirmação de alguns sinais encontrados no espectro de RMN de ${ }^{1} \mathrm{H}$ foram adicionadas algumas substâncias puras, como cafeína, catecol, trigonelina, ácidos cafeico, ferúlico, quínico e 5cafeoilquínico.

\section{Quantificação do teor de cafeína}

Para as análises quantitativas as amostras foram preparadas de três formas distintas:

Amostras A1 - A13 - Foram preparadas em uma cafeteira do tipo expresso, utilizando-se 10,0 g de pó de café e 72,0 $\mathrm{mL}$ de água a uma temperatura de $95{ }^{\circ} \mathrm{C}$. Para as medidas de RMN utilizou-se uma mistura de 5,0 $\mathrm{mL}$ do cafezinho, obtido imediatamente após o preparo, e 2,0 mL do padrão interno ( $N, N$-dimetilformamida $947 \mathrm{mg} / \mathrm{L}) \mathrm{e}$, em uma alíquota de 0,6 $\mathrm{mL}$ dessa mistura, adicionaram-se duas gotas de DMSO-d 6 para ajuste da homogeneidade do campo magnético.

Amostra A14 - Café solúvel e descafeinado, foi preparada a partir de 3,0 g de café solúvel comercial e adição de 60,0 mL de água fervente. Para a execução das medidas de RMN, e posterior quantificação do teor da cafeína, o cafezinho assim obtido foi misturado com uma solução do padrão interno, conforme descrito para as amostras de A1-A13.

Amostras B1 - B20 - Foram coletadas no comércio local, São Carlos-SP, e, após aproximadamente $4 \mathrm{~h}$, as amostras foram submetidas às análises via RMN conforme descrito para as amostras de A1-A13. Estas receberam duas classificações de acordo com a forma de preparo: de B1 a B9 como café caseiro e de B10 a B20, café expresso.
Após a aquisição e o processamento dos espectros de hidrogênio, a área do pico em 7,9 ppm, referente ao hidrogênio pirrólico da cafeína, foi relacionado com a área do pico do padrão interno em 8,1 ppm, hidrogênio carbonílico. Este procedimento, juntamente com a construção de uma curva de calibração, possibilitou a determinação do teor de cafeína.

\section{Validação do método}

A repetibilidade do método foi avaliada através dos valores do desvio padrão, sendo que para os cafés preparados no laboratório foram realizadas 15 medidas e para as bebidas coletadas no comércio e do café descafeinado, efetuaram-se 5 medidas para cada uma.

O teste de reprodutibilidade foi obtido através da execução das mesmas medidas, inclusive o preparo da amostra, por um analista diferente. Foi analisada em quintuplicata uma das amostras após ter decorrido aproximadamente 4 meses da primeira medida.

O limite de quantificação foi calculado a partir do teor de cafeína encontrado em amostras de cafés descafeinados.

O limite de detecção foi estimado adquirindo-se espectros de $\mathrm{RMN}$ de ${ }^{1} \mathrm{H}$ de soluções aquosas de cafeína com diluições decrescentes.

A exatidão foi calculada através da diferença entre duas medidas da concentração de cafeína na bebida. A primeira medida foi feita utilizando-se o café descafeinado sem adição de cafeína, e a segunda, adicionando-se uma quantidade conhecida à mesma amostra. Desta forma obtivemos o valor experimental, extraído da integração do sinal, que foi comparado com valor da quantidade de cafeína adicionada.

\section{RESULTADOS E DISCUSSÃO}

A eficiência da sequiência de pulsos compostos, $\operatorname{zgcppr}^{30,31}$, utilizada na aquisição dos espectros de $\mathrm{RMN}$ de ${ }^{1} \mathrm{H}$, que pode ser verificada na Figura 1, mostra os sinais de interesse com uma boa relação sinal/ruído e sem causar perda dos sinais próximos à irradiação. A eficiência na supressão do sinal da água foi importante tanto para as análises quantitativas, no que se refere a afetar ao mínimo a intensidade dos sinais e, conseqüentemente, os valores das suas integrais, como também na identificação dos compostos, garantindo que nenhum sinal fosse suprimido.

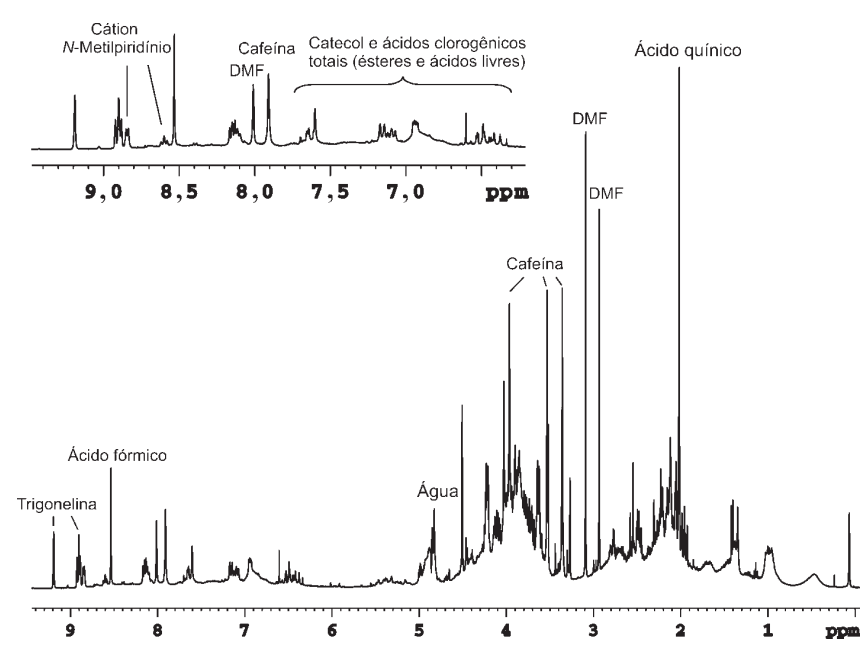

Figura 1. Espectro de RMN de ${ }^{l} \mathrm{H}$ obtido diretamente da bebida de café usando-se a seqüência de pulsos compostos (zgcppr) para a supressão do sinal da água 


\section{Identificação dos constituintes majoritários}

As estruturas dos compostos identificados neste trabalho são mostradas na Figura 2, e os valores dos deslocamentos químicos de ${ }^{1} \mathrm{H}$ e ${ }^{13} \mathrm{C}$ estão apresentados nas Tabelas $1 \mathrm{e} 2$, respectivamente.

$\mathrm{O}$ experimento de 2D homonuclear, gCOSY, foi executado com supressão do sinal dos hidrogênios da água para garantir uma melhor relação sinal/ruído. A partir dele foi possível observarmos as seguintes correlações: trigonelina - $\mathrm{H} 5$ com H4 e H6, e H2 com H4 e H6; $N$-metilpiridina - H2 e H6 com H3 e H5 e também de H3 e H5 com H4; ácidos cafeico/ferúlico/clorogênicos (todos na mesma região) - H7 com H8 e ácido quínico - H3 com H4, H5 com H6 (equatorial e axial) e também $\mathrm{H} 3$ com $\mathrm{H} 2$ (equatorial e axial).

Os espectros de gHSQC e gHMBC foram usados para a determinação dos deslocamentos químicos de ${ }^{13} \mathrm{C}$. Utilizando o experimento de gHSQC, foi possível atribuir os valores dos deslocamen-<smiles>Cn1c(=O)c2c(ncn2C)n(C)c1=O</smiles>

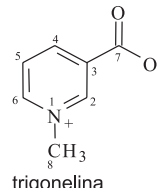<smiles>CCCCCCOC=O</smiles><smiles>Oc1ccccc1O</smiles>

catecol

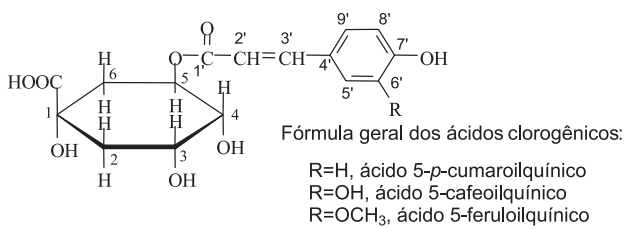

Figura 2. Estruturas dos compostos identificados na bebida de café

Tabela 1. Deslocamentos químicos $\delta(\mathrm{ppm})$ de $\mathrm{RMN}$ de ${ }^{1} \mathrm{H}$, constante de acoplamento $\mathrm{J}(\mathrm{Hz})$ e correlações via gHMBC para os compostos identificados na bebida de café

\begin{tabular}{|c|c|c|c|c|c|c|c|c|c|}
\hline \multirow[t]{2}{*}{$\mathrm{n}^{\circ}$. } & \multicolumn{9}{|c|}{ substâncias identificadas } \\
\hline & Cafeína & Trigonelina & $N$-Metilpiridina & Catecol & $\begin{array}{l}\text { Ácido } \\
\text { quínico }\end{array}$ & $\begin{array}{l}\text { Ácido } \\
\text { cafeico }\end{array}$ & $\begin{array}{l}\text { Ácido } \\
\text { ferúlico }\end{array}$ & $\begin{array}{c}\text { Ácido } \\
\text { clorogênico }\end{array}$ & $\begin{array}{l}\text { Ácido } \\
\text { fórmico }\end{array}$ \\
\hline $\mathrm{H} 2$ & - & $\begin{array}{c}9,18 \mathrm{~s} \\
* 146,0 ; * 49,2\end{array}$ & $\begin{array}{c}8,83 \mathrm{~d} \ell(6,4 \mathrm{~Hz}) \\
* 128,5 ; * 49,2\end{array}$ & - & $\begin{array}{c}1,90-2,16 \mathrm{~m} \\
* 179,5\end{array}$ & $\begin{array}{c}7,17-7,21 \mathrm{~m} \\
\mathrm{~d}(2,6 \mathrm{~Hz})\end{array}$ & $\begin{array}{c}\text { 7,17- 7,21 m } \\
\mathrm{d}(2,6 \mathrm{~Hz})\end{array}$ & $\begin{array}{c}1,90-2,16 \mathrm{~m} \\
\quad * 179,5\end{array}$ & - \\
\hline $\mathrm{H} 3$ & - & - & $8,12 \mathrm{dd} \ell(7,5 ; 6,4 \mathrm{~Hz})$ & $\begin{array}{c}6,96 \\
\mathrm{~d}(8,3 \mathrm{~Hz}) \\
* 145,8\end{array}$ & 4,09 & - & - & 4,09 & - \\
\hline $\mathrm{H} 4$ & - & $\begin{array}{c}8,88 \mathrm{~d} \ell(6,2 \mathrm{~Hz}) \\
* 168,0 ; * 146,8\end{array}$ & $\begin{array}{c}8,60 \mathrm{te}(7,5 ; 7,5 \mathrm{~Hz}) \\
* 128,5\end{array}$ & $\begin{array}{c}6,97 \\
\mathrm{~d}(8,3 \mathrm{~Hz}) \\
* 145,8\end{array}$ & 3,62 & - & - & 3,62 & - \\
\hline H5 & - & $\begin{array}{c}8,15 \\
\text { dd }(7,6 ; 6,2 \mathrm{~Hz})\end{array}$ & $\begin{array}{c}8,12 \\
\mathrm{dd} \ell(7,5 ; 6,4 \mathrm{~Hz})\end{array}$ & $\begin{array}{c}6,97 \\
\mathrm{~d}(8,3 \mathrm{~Hz}) \\
* 145,8\end{array}$ & 4,20 & $\begin{array}{c}6,96 \mathrm{~d} \\
(8,3 \mathrm{~Hz})\end{array}$ & $\begin{array}{c}6,96 \mathrm{~d} \\
(8,3 \mathrm{~Hz})\end{array}$ & - & - \\
\hline $\mathrm{H} 8$ & $\begin{array}{c}7,89 \\
* 149,8 \\
* 108,0\end{array}$ & $4,49 \mathrm{~s}$ & - & - & - & $\begin{array}{c}7,59-7,73 \mathrm{~d} \\
(15,8 \mathrm{~Hz}) \\
* 170,0\end{array}$ & $\begin{array}{c}7,59-7,73 \mathrm{~d} \\
(15,8 \mathrm{~Hz}) \\
* 170,0\end{array}$ & - & - \\
\hline H10 & $\begin{array}{c}3,48 \\
* 153,8 \\
* 149,8\end{array}$ & - & - & - & - & - & - & - & - \\
\hline H11 & $\begin{array}{c}3,30 \\
* 153,8 \\
* 158,0\end{array}$ & - & - & - & - & - & - & - & - \\
\hline H12 & $\begin{array}{c}3,91 \\
* 108,0 \\
* 144,8\end{array}$ & - & - & - & - & - & - & - & - \\
\hline H9' & - & - & - & - & - & - & - & $7,09-7,16 \mathrm{~m}$ & - \\
\hline $\mathrm{OCH}_{3}$ & - & - & - & - & - & - & 3,84 & - & - \\
\hline
\end{tabular}

* deslocamentos químicos obtidos via gHMBC; s - singleto; $\mathrm{d}$ - dubleto; dl - dubleto largo; ddl - duplo dubleto largo; tl - tripleto largo; $\mathrm{m}$ - multipleto. 
Tabela 2. Deslocamentos químicos $\delta(\mathrm{ppm})$ de $\mathrm{RMN}$ de ${ }^{13} \mathrm{C}$ dos compostos identificados na bebida de café

\begin{tabular}{|c|c|c|c|c|c|c|c|c|c|}
\hline \multirow[t]{2}{*}{$\mathrm{n}^{\circ}$. } & \multicolumn{9}{|c|}{ substâncias identificadas } \\
\hline & Cafeína & Trigonelina & $N$-Metilpiridina & Catecol & $\begin{array}{l}\text { Ácido } \\
\text { quínico }\end{array}$ & $\begin{array}{l}\text { Ácido } \\
\text { cafeico }\end{array}$ & $\begin{array}{l}\text { Ácido } \\
\text { ferúlico }\end{array}$ & $\begin{array}{c}\text { Ácido } \\
\text { clorogênico }\end{array}$ & $\begin{array}{l}\text { Ácido } \\
\text { fórmico }\end{array}$ \\
\hline $\mathrm{C} 1$ & - & - & - & $* 145,8$ & 76,0 & $* 128,0$ & $* 128,0$ & 76,0 & 171,9 \\
\hline $\mathrm{C} 2$ & $* 153,8$ & 146,8 & 145,9 & $* 145,8$ & 39,0 & 115,7 & 115,7 & 39,0 & - \\
\hline $\mathrm{C} 3$ & - & $* 146,0$ & 128,5 & 116,9 & 69,3 & 149,0 & - & 69,3 & - \\
\hline $\mathrm{C} 4$ & $* 149,8$ & 146,5 & 146,4 & 123,4 & 76,5 & 147,2 & 147,2 & 76,5 & - \\
\hline $\mathrm{C} 5$ & $* 108,0$ & 128,5 & 128,5 & 123,4 & 71,1 & 117,0 & 117,0 & 71,1 & - \\
\hline C6 & $* 158,0$ & 145,5 & 145,9 & 116,9 & 39,0 & 123,4 & 123,4 & 39,0 & - \\
\hline $\mathrm{C} 7$ & - & 168,0 & 49,2 & - & $* 179,5$ & 115,6 & 115,6 & $* 179,5$ & - \\
\hline $\mathrm{C} 8$ & 144,8 & $* 49,2$ & - & - & - & 146,8 & 146,8 & - & - \\
\hline C9 & - & - & - & - & - & $* 170,0$ & $* 170,0$ & - & - \\
\hline $\mathrm{C} 10$ & 30,9 & - & - & - & - & - & - & - & - \\
\hline $\mathrm{C} 11$ & 28,9 & - & - & - & - & - & - & - & - \\
\hline $\mathrm{C} 12$ & 34,5 & - & - & - & - & - & - & - & - \\
\hline $\mathrm{C} 2^{\prime}$ & - & - & - & - & - & - & - & 146,8 & - \\
\hline C3' & - & - & - & - & - & - & - & 115,6 & - \\
\hline $\mathrm{C} 5$ & - & - & - & - & - & - & - & 115,7 & - \\
\hline C6' & - & - & - & - & - & - & - & 149,0 & - \\
\hline C7' & - & - & - & - & - & - & - & 147,2 & - \\
\hline C8' & - & - & - & - & - & - & - & 117,0 & - \\
\hline C9' & - & - & - & - & - & - & - & 123,4 & - \\
\hline $\mathrm{OCH}_{3}$ & - & - & - & - & - & - & 65,5 & - & - \\
\hline
\end{tabular}

* correlações obtidas via gHMBC

tos químicos dos carbonos via ${ }^{1} \mathrm{~J}_{1 \mathrm{H}-13 \mathrm{C}}$ e com o experimento gHMBC atribuiu-se todos os demais sinais através das correlações ${ }^{\mathrm{n}} \mathrm{J}_{1 \mathrm{H}-13 \mathrm{C}}$, que são mostrados na Tabela 2.

\section{Quantificação do teor de cafeína}

Para cálculo do teor de cafeína, montou-se a curva de calibração mostrada na Figura 3, obtendo-se os valores encontrados para as amostras de cafés preparadas no laboratório sob condições controladas e para as amostras coletadas no comércio local, cujos resultados são apresentados nas Tabelas 3 e 4, respectivamente. Nelas podemos observar que existe uma grande variação tanto para as amostras de cafés produzidas no laboratório como, e principalmente, nas amostras encontradas no comércio local.

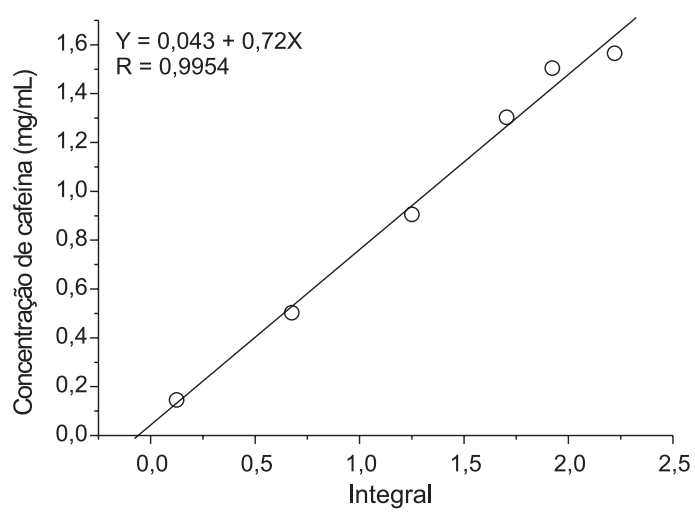

Figura 3. Curva de calibração para o teor de cafeína

Para as amostras de cafés preparadas em laboratório, os valores variaram de 49,8 a 69,6 mg/60 mL (volume aproximado de uma xícara de café utilizada no comércio em geral). Essa variação pode estar associada ao ano de colheita, à origem geográfica da planta, às espécies e/ou cultivares utilizadas, às diferentes composições das
Tabela 3. Teor de cafeína encontrado nos cafés preparados em laboratório

\begin{tabular}{llc}
\hline Amostras & Tipo de café & $\begin{array}{c}\text { Concentração de cafeína } \\
\mathrm{mg} / 60 \mathrm{~mL}\end{array}$ \\
\hline A1 & Expresso & $69,6 \pm 1,8$ \\
A2 & Extra-Forte & $64,8 \pm 6,6$ \\
A3 & Extra-Forte & $63,6 \pm 5,4$ \\
A4 & Forte & $58,8 \pm 4,8$ \\
A5 & Tradicional & $55,8 \pm 4,2$ \\
A6 & Orgânico & $54,6 \pm 4,2$ \\
A7 & Tradicional & $52,2 \pm 3,0$ \\
A8 & Suave & $51,6 \pm 4,2$ \\
A9 & Extra-Forte & $51,6 \pm 2,4$ \\
A10 & Forte & $51,0 \pm 1,2$ \\
A11 & Forte & $51,0 \pm 4,2$ \\
A12 & Forte & $50,4 \pm 1,2$ \\
A13 & Exportação & $49,8 \pm 7,2$ \\
A14 & Descafeinado & $7,2 \pm 1,2$ \\
\hline
\end{tabular}

* corresponde a aproximadamente uma xícara de café

espécies na mistura, à granulometria dos pós e ao grau de torra ${ }^{8,32,33}$. Nas amostras de cafezinho coletadas no comércio, encontrou-se uma variação ainda maior, sua concentração variou de 29,4 a 79,8 mg/60 $\mathrm{mL}$, já que nestas não houve controle algum tanto quanto aos produtos como no procedimento utilizado no preparo da bebida.

Considerando-se que o consumo de cafeína em doses moderadas, aproximadamente $300 \mathrm{mg} /$ dia, não deve causar danos à saúde de um indivíduo normal, e que o consumo exagerado pode estar relacionado com o possível desenvolvimento de algumas doenças, como hipertensão, úlceras, taquicardia, etc ${ }^{34}$, os resultados obtidos demonstram que o controle diário baseado em xícaras de cafés não é uma medida recomendada. Dependendo dos vários fatores apontados acima, poder-se-ia estar consumindo mais ou menos cafeína 
Tabela 4. Teor de cafeína encontrado nos cafés coletados no comércio local (São Carlos-SP)

\begin{tabular}{llc}
\hline Amostras & Tipo de café & $\begin{array}{c}\text { Concentração de cafeína } \\
\mathrm{mg} / 60 \mathrm{~mL}\end{array}$ \\
\hline B1 & Caseiro & $61,8 \pm 4,2$ \\
B2 & Caseiro & $52,2 \pm 1,2$ \\
B3 & Caseiro & $51,0 \pm 1,2$ \\
B4 & Caseiro & $49,2 \pm 1,2$ \\
B5 & Caseiro & $45,6 \pm 0,6$ \\
B6 & Caseiro & $40,2 \pm 1,8$ \\
B7 & Caseiro & $40,2 \pm 1,2$ \\
B8 & Caseiro & $37,8 \pm 1,2$ \\
B9 & Caseiro & $29,4 \pm 0,6$ \\
B10 & Expresso & $79,8 \pm 1,2$ \\
B11 & Expresso & $69,0 \pm 1,8$ \\
B12 & Expresso & $64,8 \pm 1,2$ \\
B13 & Expresso & $58,8 \pm 0,6$ \\
B14 & Expresso & $55,8 \pm 1,8$ \\
B15 & Expresso & $50,4 \pm 1,2$ \\
B16 & Expresso & $49,8 \pm 1,2$ \\
B17 & Expresso & $48,0 \pm 1,2$ \\
B18 & Expresso & $46,2 \pm 0,6$ \\
B19 & Expresso & $41,4 \pm 0,6$ \\
B20 & Expresso & $40,2 \pm 0,6$ \\
\hline
\end{tabular}

* corresponde a aproximadamente uma xícara de café

diariamente em função do teor de cafeína encontrado em cada tipo de bebida e não, no número de xícaras consumidas.

Os teores de cafeína encontrados nas amostras estudadas estão em concordância com os dados da literatura ${ }^{33}$ baseados na cromatografia líquida de alta eficiência (CLAE). Nos dois métodos foi observado um teor médio de cafeína, para os cafés do tipo expresso, um pouco superior aos do tipo caseiro. No entanto, algumas bebidas caseiras apresentaram valores superiores aos cafés expressos (Tabela 4). Isso é justificável uma vez que as amostras foram coletadas no comércio da cidade e não tiveram nenhum controle do seu preparo.

Com relação à amostra de café instantâneo e descafeinado, A14, observa-se um resíduo de cafeína da ordem de $0,2 \%$. Esse valor está em concordância com a quantidade máxima de cafeína, em cafés descafeinados, estabelecida pelas normas de qualidade da Comunidade Européia ${ }^{8}$.

Para validação do método via $\mathrm{RMN}$ de ${ }^{1} \mathrm{H}$ encontrou-se boa reprodutibilidade e repetibilidade, que pode ser observada nos desvios padrões das Tabelas 3 e 4, exatidão de 95,5\%, limite de quantificação de $0,12 \mathrm{mg} / \mathrm{mL}$ e limite de detecção de $0,027 \mathrm{mg} / \mathrm{mL}$.

\section{CONCLUSÕES}

Os experimentos de RMN 1D e 2D, auxiliados pela adição das substâncias padrões, permitiram a identificação dos constituintes majoritários no cafezinho como cafeína, trigonelina, $\mathrm{N}$-metilpiridina, catecol, ácido fórmico e os ácidos quínico, cafeico, ferúlico e clorogênicos, sem o seu isolamento prévio. Quanto à determinação do teor de cafeína na bebida, a técnica de RMN mostrou ser uma ferramenta útil e confiável, com boa repetibilidade e reprodutibilidade entre as medidas, e uma exatidão de $95,5 \%$.

\section{AGRADECIMENTOS}

Ao CNPq e CAPES pelas bolsas de estudos e à FAPESP pelo apoio financeiro. Ao Prof. Dr. P. Mazzafesa, Departamento de Fisiologia Vegetal, IB-Unicamp, por ceder algumas substâncias puras utilizadas como padrões.

\section{REFERÊNCIAS}

1. Galli, V.; Barbas, C.; J. Chromatogr., A 2004,1032, 299.

2. Saldaña, M. D. A.; Mazzafera, P.; Mohamed, R.; Ciência e Tecnologia de Alimentos 1997, 17, 371

3. Navarini, L.; Gilli, R.; Gombac, V.; Abatangelo, A.; Bosco, M.; Toffanin, R.; Carbohydr. Polym. 1999, 40, 71.

4. Schenker, S.; Heinemann, C.; Huber, M.; Pompizzi, R.; Perren, R.; Escher, F.; J. Food Sci. 2002, 67, 60.

5. Roos, B.; Weg, G. V. D.; Urgert, R.; Bovenkamp, P. V.; Charrier, A.; Katan, M. B.; J. Agric. Food Chem. 1997, 45, 3065.

6. Dutra, E. R.; Oliveira, L. S.; Franca, A. S.; Ferraz, V. P.; Afonso, R. J. C. F.; J. Food Eng. 2001, 47, 241.

7. Martin, M. J.; Pablos, F.; González, A. G.; Talanta 1998, 46, 1259.

8. Clarke, R.J.; Macrae, R.; Coffee: Chemistry, Elsevier Applied Science Publishers LTD: London, 1985.

9. Carvalho, V. D.; Chalfoun, S. M.; Informe Agropecuário 1985, 11, 79.

10. Carvalho, V. D.; Chagas, S. J. R.; Chalfoun, S. M.; Informe Agropecuário 1997, 18,5 .

11. Monteiro, M. C.; Trugo, L. C.; Quim. Nova 2005, $28,637$.

12. De Maria, C. A. B.; Trugo, L. C.; Aquino Neto, F. R.; Moreira, R. F. A.; Alviano, C. S.; Food Chem. 1998, 33, 203.

13. De Maria, C. A. B.; Moreira, R. F. A.; Trugo, L. C.; Quim. Nova 1999, 22, 209.

14. Mattila, P.; Kumpulainen, J.; J. Agric. Food Chem. 2002, 50, 3660.

15. Del Castillo, M. D.; Ames, J. M.; Gordon, M. H.; J. Agric. Food Chem. 2002, 50, 3698.

16. Moreira, R. F. A.; De Maria, C. A. B.; Trugo, L. C.; Quim. Nova 2000, 23, 195.

17. Del Castillo, M. L. R.; Herraiz, M.; Blanch, G.; J. Agric. Food Chem. 1999, 47, 1525.

18. Bouhsain, Z.; Garrigues, J. M.; Garrigues, S.; Guardia, M.; Vib. Spectrosc. 1999, 21,143

19. Briandet, R.; Kemsley, E. K.; Wilson, R. H.; J. Agric. Food Chem. 1996, 44, 170.

20. Esteban-Díez, I.; González-Sáiz, J. M.; Pizarro, C.; Anal. Chim. Acta 2004, $514,57$.

21. Carrera, F.; León-Camacho, M.; Pablos, F.; González, A. G.; Anal Chim. Acta 1998, 370, 131

22. Bicchi, C. P.; Panero, O. M.; Pellegrino, G. M.; Vanni, A.C.; J. Agric. Food Chem. 1997, 45, 4680.

23. Maeztu, L.; Sanz, C.; Andueza, S.; De Peña, M. P.; Bello, J.; Cid, C.; J. Agric. Food Chem. 2001, 49, 5437.

24. Bosco, M.; Toffanin, R.; De Palo, D.; Zatti, L.; Segre, A.; J. Sci. Food Agric. 1999, 79, 869.

25. Charlton, A. J.; Farrington, W. H. H.; Brereton, P.; J. Agric. Food Chem. 2002, 50, 3098.

26. D’Agostina, A.; Boschin, G.; Bacchini, F.; Arnoldi, A.; J. Agric. Food Chem. 2004, 52, 7118 .

27. Stadler, R. H.; Varga, N.; Hau, J.; Vera, F. A.; Welti, D. H.; J. Agric. Food Chem. 2002, 50, 1192.

28. Stadler, R. H.; Varga, N.; Milo, C.; Schilter, B.; Vera, F. A.; Welti, D. H.; J. Agric. Food Chem. 2002, 50, 1200.

29. Weckerle, B.; Gáti, T.; Tóth, G.; Schreier, P.; Phytochemistry 2002, 60, 409.

30. Claridge, T. D. W.; High-Resolution NMR Techniques in Organic Chemistry, Pergamon: Oxford, 1999.

31. Bax, A. D.; J. Magn. Reson. 1985, 65, 142.

32. Andrade, P. B.; Leitão, R.; Seabra, R. M.; Oliveira, M. B.; Ferreira, M. A.; Food Chem. 1998, 61, 511.

33. Camargo, M. C. R.; Toledo, M. C. F.; Ciência e Tecnologia de Alimentos 1998, 18,421

34. http://www.ific.org/publications/reviews/caffeineir.cfm, acessada em Outubro 2005 